conditions such as HIV/AIDS, tuberculosis, diabetes, hypertension, family planning and asthma. Available records indicated that FSW still patronised 14 of these clinics. Overall, there has been marginal increase in the FSW attendance compared with statistics during the time of the project.

Conclusion The integration of the STI intervention project into the primary level of care in the Ghana Health Services is a success. FSW like other clients who patronise health facilities deserve equal dignity, respect and quality care.

Disclosure of interest statement No conflict of interest.

\section{P12.14 CLINICAL DESCRIPTIONS OF PCR POSITIVE EARLY SYPHILIS INFECTIONS}

${ }^{1} \mathrm{JM}$ Towns*, ${ }^{2} \mathrm{DE}$ Leslie, ${ }^{1}$ I Denham, ${ }^{2} \mathrm{~F}$ Azzato, ${ }^{1,3} \mathrm{CK}$ Fairley, ${ }^{1,3} \mathrm{MY}$ Chen. ${ }^{1}$ Melbourne Sexual Health Centre (MSHC), Alfred Health; ${ }^{2}$ Victorian Infectious Diseases Reference Laboratory (VIDRL), Doherty Institute; ${ }^{3}$ Central Clinical School, Monash University

\subsection{6/sextrans-2015-052270.494}

Background Globally, syphilis remains a major and resurgent public health problem with high incidence rates in many settings, including among men who have sex with men (MSM). Left untreated, syphilis can lead to further transmission, morbidity and enhanced Human Immunodeficiency Virus (HIV) transmission. A primary chancre is classically described as an indurated single painless ulcer at the site of Treponema pallidum inoculation. However, recent clinical experience is that primary syphilis can present atypically, as multiple and/or painful ulcers with features suggestive of genital herpes. We aimed to describe serology and Treponema pallidum polymerase chain reaction (Tp PCR) positive lesions of primary syphilis in men, the rates of painful or multiple lesions, whether there was concurrent genital Herpes simplex virus (HSV) infection and whether concurrent HIV infection altered the presentation.

Methods Tp PCR positive results with confirmatory syphilis serology and HSV PCR results reported by VIDRL were identified and compared to MSHC medical records over a five-year period from 2010 to 2014.

Results 183 patients fulfilled the criteria of Tp PCR positive primary syphilis. Primary syphilis lesions were frequently painful $(49.2 \%)$ or multiple $(37.7 \%)$, and were infrequently associated with HSV (2.7\%). Presentation was not significantly altered by HIV status. Anal lesions were more common in HIV positive men $(34.2 \%)$ than in HIV negative men (11.6\%). Syphilis reinfections were more common in HIV positive men (39.5\%) than in HIV negative men (11.7\%).

Conclusion Tp PCR is a useful tool to confirm syphilis as a cause of genital lesions and positivity may precede serological markers. Early syphilis lesions may be clinically misidentified as HSV infection if syphilis is not considered. Awareness of the clinical variability of primary syphilis lesions should be included in health promotion messages to the public and health care providers.

Disclosure statement There are no conflicts of interest.

\section{P12.15 PENILE PAPULONECROTIC TUBERCULOSIS: IS IT A SEXUALLY TRANSMITTED DISEASE?}

Gupta Somesh*, R Bhatia. Department of Dermatology \& Venereology, All India Institute of Medical Sciences, New Delhi, India

10.1136/sextrans-2015-052270.495
Introduction Ulcertaive lesions of tuberculosis on penis have been classified as papulonecrotic tuberculids, though clinical presentation and absence of generalised involvement suggest that it is sexually transmitted inoculation tuberculosis.

Methods All patients with penile papulonecrotic tuberculosis (PPNT) presented during past 5 years and their wives were investigated for pulmonary and extra-pulmonary tuberculosis including reproductive tract tuberculosis (RTB). All histopathologically diagnosed patients were treated with anti-tubercular therapy (ATT) and followed up.

Results Seven patients, aged between 21-30 years, all married, were diagnosed with PPNT based on caseating epithelioid cell granulomas in histopathology. They presented with recurrent genital ulcers for the duration ranging from 3-8 years. These would start as asymptomatic papulopustules over the glans penis/prepuce which would breakdown to form painful ulcers in 1-2 weeks and heal with scarring in another 2-3 weeks. Five patients were diagnosed in the past as genital herpes due to episodic nature and received suppressive antiviral therapy without any response. All the patients denied any premarital or extra marital sexual contact or oral insertive sex. A dramatic response was seen in PPNT lesions within 4-8 weeks of starting ATT.

There was a history of primary infertility due to RTB in the spouses of two patients. Spouse of one patient conceived after a course of ATT.

Mantoux test was strongly positive in all, and PCR for Mycobacterium Tuberculosis was positive in 3 patients. HIV serology and VDRL were negative. No internal focus of tuberculosis was found.

Conclusion Several features suggest that penile tuberculide may actually be sexually acquired implantation tuberculosis. The PPNT is an isolated disease without involvement of other parts. The concurrent RTB in the spouse raises the possibility of it being sexually transmitted. With increasing acceptability of oral sex, the inoculation may take place from sputum of a patient with pulmonary TB. This hypothesis needs genotyping studies to confirm.

Disclosure of interest statement None.

\section{P12.16 OLDER PATIENTS ATTENDING SEXUALLY TRANSMITTED INFECTIONS CLINICS}

${ }^{1}$ SA Tuddenham*, ${ }^{1} \mathrm{KR}$ Page, ${ }^{2} \mathrm{CP}$ Chaulk, ${ }^{1} \mathrm{KG}$ Ghanem. ${ }^{1}$ Johns Hopkins University School of Medicine; ${ }^{2}$ Baltimore City Health Department, Baltimore, MD, USA

\subsection{6/sextrans-2015-052270.496}

Background Many older patients remain sexually active well into their eighth decade of life. Surveillance data suggest that rates of sexually transmitted infections (STIs) in this age group may be increasing. We sought to compare demographics, risk behaviours and predictors of acute infections in patients 50 years and older versus younger patients attending STI clinics in Baltimore, Maryland.

Methods Retrospective study from a large electronic database of all visits to two urban STI clinics between 2005 and 2010. Proportions were compared using the $c^{2}$ test. Logistic regression was used to assess predictors of acute STIs in older versus younger groups.

Results 4461 first visits for patients over 50 and 4893 visits for patients under 50 were included in the analysis. Patients over 50 frequently reported high-risk behaviours $[35.4 \%$ (CI $0.34-0.37$ ) vs. $52.7 \%$ (CI $0.51-0.54$ ) in those $<50$ ] but they were more 
likely than younger patients to report never using condoms [32.6\% (CI $0.31-0.34$ ) vs. $24.1 \%$ (CI $0.23-0.25)$ ]. The overall prevalence of acute STIs was $18.1 \%$ (CI $0.17-0.19$ ) in older patients and $25.8 \%$ (CI $0.25-0.27$ ) in younger patients. Older women were more likely to be diagnosed with trichomoniasis [21.5\% of those tested (CI 18.6-24.5) vs. $13.1 \%$ (CI $11.5-$ 14.8)]. Black race was predictive of having an acute STI in younger men [OR 2.2 (CI 1.47-3.35)] and women [OR 2.7 (CI 1.34-5.30)] but not in older men [OR 1.2 (CI 0.79-1.73)] or women [OR 1.2 (CI 0.43-3.15)].

Conclusion Older patients who seek care at STI clinics engage in significant risk behaviours. Race, a factor strongly predictive of acute STIs in young men and women is not a significant predictor of STIs in older persons.

Disclosure of interest statement No sources of funding or conflicts of interest to disclose.

\section{P12.17 SHOULD SPECILIAST SEXUAL HEALTH SERVICES STILL SEE SELF-REFERRALS}

${ }^{1}$ A Robertson*, ${ }^{2}$ A Carswell. ${ }^{1}$ MidCentral Health District Health Board, NZ; ${ }^{2}$ University of Otago, NZ

\subsection{6/sextrans-2015-052270.497}

Introduction There is an international trend in the re-development of specialist sexual health services as referral only services or with a reduced self-referral component. This study evaluates the value of retaining a self-referral component to service provision.

Methods An audit of attendance reasons to a provincial sexual health service for 354 episodes of care from 2012-2013 were analysed.

Data collected for national STI surveillance were analysed to compare the age and gender of patients treated for bacterial STIs in 2013 through the sexual health service with those treated by other providers.

Further analysis will be undertaken to assess the characteristics of patients attending a specialist service for STI management and trends with time.

Results Confidentiality is the most common reason for attending a specialist sexual health service (35\%) followed by cost (29\%).

$35 \%$ of gonorrhoea cases and $20 \%$ of chlamydia cases in the region are managed through the sexual health service. However $35 \%$ of men with chlamydia are managed through the service and $56 \%$ of those over 30 .

Conclusion Despite youth health services and general practices seeing an increasing number of young people for chlamydia management, specialist services continue to see a concentrated group of patients with bacterial STIs to provide opportunities for training and to gain knowledge of local sexual networks.

Disclosure of interest statement The work of Alex Carswell was sponsored by the Palmerston North Undergraduate Medical education Trust. No pharmaceutical grants were received in the development of this study.

\section{P12.18 SEXUAL AND REPRODUCTIVE HEALTH IN RURAL VICTORIA: WHAT URGENT CARE SERVICES ARE AVAILABLE AND ARE THEY ADEQUATE?}

${ }^{1} \mathrm{CC}$ Morton*, 1,2JE Tomnay, 'SD Kauer, ${ }^{1} \mathrm{JG}$ Walker. 'Department of General Practice, Melbourne Medical School, University of Melbourne; ${ }^{2}$ Centre for Excellence in Rural Sexual Health, Rural Health Academic Centre, University of Melbourne

\subsection{6/sextrans-2015-052270.498}

Introduction Strong evidence exists that young, rural people face significant barriers to accessing sexual and reproductive health (SRH) services. What influence this has on chlamydia notifications is unknown. Regional hospital urgent care services have the potential to provide after-hours SRH services but little is known about their capacity, policies and procedures in Victoria. This project aims to investigate the availability of testing for sexually transmissible infections (STIs) and provision of emergency contraception (EC) in regional hospitals and explore any relationships with chlamydia notifications.

Methods All urgent care and regional trauma services $(\mathrm{N}=60)$ were surveyed by phone. Cross-sectional data were collected to determine policies and practices for STI testing and EC provision. Descriptive analysis was conducted by geographical region and an analysis will be performed to determine any relationship between access to after-hours SRH services and chlamydia notifications by region.

Results To date, 41/60 (68\%) hospitals have provided data. 29\% knew of a policy for STI testing and all services had the capacity for STI testing but 29\% reported that they would refer patients for STI testing elsewhere. The majority of services $(66 \%)$ had EC available on site. Of the 13 services that didn't provide EC, only 1 could refer to another 24 -hr service within 30 min drive. Of the hospitals that would refer to the nearest regional centre hospital, all were $>30 \mathrm{~min}$ away and not accessible by public transport. EC provision varied widely between regions (range 29-83\%). Further analysis between chlamydia notifications and STI testing availability will be explored.

Conclusion The data collected demonstrated the heterogeneity of STI testing and EC available to young people after-hours in regional areas. The bulk of services are offered at the discretion of individual hospitals. Accessibility, affordability, availability, accommodation and acceptability continue to be obstacles for young people in regional areas in rural Victoria.

Disclosure of interest statement The authors declare that they have no competing interests.

\section{P13 - Operational and health systems research}

\section{P13.01 TOWARDS UNIVERSAL ACCESS: THE PAPUA NEW GUINEA (PNG) COMPANION PRODUCT CONDOM DISTRIBUTION TRIAL}

R Nixon*. Social Science Dimensions

10.1136/sextrans-2015-052270.499 